\title{
Assisted computer and imaging system improve accuracy of breast tumor size assessment after neoadjuvant chemotherapy
}

\author{
Meng Zhang, Yanqi Ma, Cuizhi Geng, Yueping Liu \\ Department of Pathology, The Fourth Hospital of Hebei Medical University, Shijiazhuang, China \\ Contributions: (I) Conception and design: M Zhang; (II) Administrative support: Y Liu, C Geng; (III) Provision of study materials or patients: M \\ Zhang; (IV) Collection and assembly of data: Y Ma; (V) Data analysis and interpretation: M Zhang; (VI) Manuscript writing: All authors; (VII) Final \\ approval of manuscript: All authors. \\ Correspondence to: Dr. Yueping Liu. Department of Pathology, the Fourth Hospital of Hebei Medical University, No. 12 Jiankang Road, Shijiazhuang \\ 050011, China. Email: annama@163.com; Dr. Cuizhi Geng. Department of Surgery, the Fourth Hospital of Hebei Medical University, No. 12 \\ Jiankang Road, Shijiazhuang 050011, China. Email: gengcuizhi@hotmail.com.
}

Background: The use of neoadjuvant therapy (NAT) in patients with early breast cancer is becoming increasingly common. The purpose of this study was to explore the combined use of breast pathology cabinet $\mathrm{X}$-ray system (CXS) to accurately assess the response to neoadjuvant treatment of breast cancer and establish a standard evaluation system.

Methods: A total of 100 patients with breast cancer after neoadjuvant treatment were randomly selected. Preoperative imaging evaluation of tumor masses were significantly degenerated, and they were randomly divided into experimental and control groups of 50 cases each. Compared with the traditional two methods of material extraction, the effective material extraction rate is comparative. Take the two largest diameters of the largest two-dimensional surface of the tumor bed as the measurement object, the macro-description value is D1/D2, the radiographic system description measurement value is the experimental group $\mathrm{d} 1 / \mathrm{d} 2$, and the correction under the microscope is worth the true size of the tumor bed $\mathrm{H} 1 / \mathrm{H} 2$ as the final test standard, calculate the difference between D1/D2 and d1/d2 with $\mathrm{H} 1$ and H2, and compare the difference between d1- H1, d2 - H2 and D1- H1, D2 - H2.

Results: The average group of tissue samples in the experimental group was 16.4, and the average group of tissue samples in the control group was 16.7, and there was no difference between the two groups; The effective tissue blocks of tumor bed samples in the experimental group were11.8, and the control group was 7.5. There is difference between the two groups. The average effective percentage of tumor bed in the experimental group was $72 \%$, and the average effective percentage of tumor bed in the control group was $44.8 \%$. The difference was also statistically significant; d1- H1, d2 - H2 and D1- H1, D2 - H2 are all different.

Conclusions: CXS assists the collection of breast tumor bed, which can significantly improve the efficiency of tumor bed collection and save the cost of collection. Compared with the maximum diameter of the tumor bed by eyes, the CXS mapping value is closer to the value measured under the microscope.

Keywords: X-ray radiography system; neoadjuvant therapy (NAT); effective rate of tumor bed retrieval

Submitted Jun 21, 2020. Accepted for publication Jan 22, 2021.

doi: $10.21037 /$ tcr-20-2373

View this article at: http://dx.doi.org/10.21037/tcr-20-2373

\section{Introduction}

Neoadjuvant therapy for breast cancer is playing an increasingly critical role in preoperative breast cancer treatment. Neoadjuvant therapy includes neoadjuvant chemotherapy (NACT), neoadjuvant targeted therapy and neoadjuvant endocrine therapy. The purpose is to 
reduce the mass and clinical stage, and to fully prepare for subsequent surgical removal of the lesion. With the rise of artificial intelligence and computer-assisted learning technology, it is widely used in the assessment of breast cancer, such as the use of convolutional neural network for automatic digital patching to restore the tumor bed, and use the three-dimensional single-cell imaging for the analysis of RNA and protein expression in intact tumour biopsies. Three-dimensional imaging technology has also been initially tried in the restoration of breast cancer bed. The morphology of the breast tumor bed after neoadjuvant treatment, the way of concentric and non-concentric contraction can be revealed in the simulated threedimensional imaging. However, changes in tumor bed have put forward new requirements for pathologist selection and pathological evaluation. Needless to say, accurate material selection and description of the tumor bed can effectively restore the true condition of the tumor bed to the greatest extent, and provide accurate data for pathological evaluation. Is there a new and effective way to determine the location of the tumor bed? Exactly describe the size? The cabinet type X-ray radiography system (CXS) was first introduced to China. This article discusses the guidance of the tumor bed material acquisition and the role of microscope bed measurement after NACT to provide a preliminary reference for tumor bed material selection. We present the following article in accordance with the MDAR checklist (available at http://dx.doi.org/10.21037/tcr-20-2373).

\section{Methods}

\section{Information}

A total of 100 breast specimens from NACT from January to September 2019 in the Fourth Hospital of Hebei Medical University were collected and randomly divided into 50 cases in the artificial group (control group) and 50 cases in the machine-assisted group (experiment group, we obtained statement confirming informed consent from all patients). The patients in both groups were females, aged 35-65 years, with a median age of 48 years. Among them, 64 patients had radical resection of breast cancer, 36 cases had breastconserving resection, and patients underwent different regimens of chemotherapy for 3-8 cycles. Complete clinical remission was achieved before. Fifty-two cases were judged as complete or near complete remission after NACT, 36 cases showed multifocal calcification on $\mathrm{X}$-ray images, and 12 cases tumors were significantly reduced to $1-2 \mathrm{~cm}$, the images of X-ray showing high-density areas, which were suspected to have residual tumor parenchymal components. Cases were screened based on the following principles: After NACT, before radical surgery, imaging tumors regressed significantly, approached or reached complete clinical remission compared to NACT. The study was conducted in accordance with the Declaration of Helsinki (as revised in 2013). The study was approved by ethics board of the Fourth Hospital of Hebei Medical University (2020K1334 ) and informed consent was taken from all the patients.

\section{Instrument}

Cabinet X-ray system.

\section{Definition}

In order to be able to simply and accurately compare the efficiency of the two material extraction methods, the efficiency of retaining bed material is defined as: the number of effective specimens (tumor parenchyma and non-tumor parenchyma but tissues with chemotherapy response)/ tumor bed specimens Number, which is the formula $\mathrm{P}=\mathrm{T} / \mathrm{N} \times 100 \%$

\section{Specimen selection and correction under the microscope}

The subjects were divided into breast-conserving specimens and radical mastectomy specimens after neoadjuvant therapy. For breast-conserving specimens, preliminary positioning was performed based on preoperative tattoos and titanium clip marks, and the specimens were irradiated with CXS for further accurate positioning. Use different colors of dye to mark the edges in different directions, and take the edges vertically. The breast was dissected at an interval of $1 \mathrm{~cm}$ and the largest two-dimensional surface of the tumor bed was exposed. The maximum diameter in both directions was described. The edges of the largest twodimensional section of the tumor bed were irradiated with $\mathrm{X}$-rays. The suspicious positive edge was recorded and the largest tumor bed was marked on the $\mathrm{X}$-ray image. The measured value of the two-dimensional surface. The largest tumor bed is obtained in order. For radical mastectomy specimens, pathologists should look for markers and preoperative image information to initially locate the tumor bed location, and expose the largest two-dimensional surface of the tumor bed at $1 \mathrm{~cm}$ intervals. According to the BIG-NABCG recommendation, for the larger tumors, 


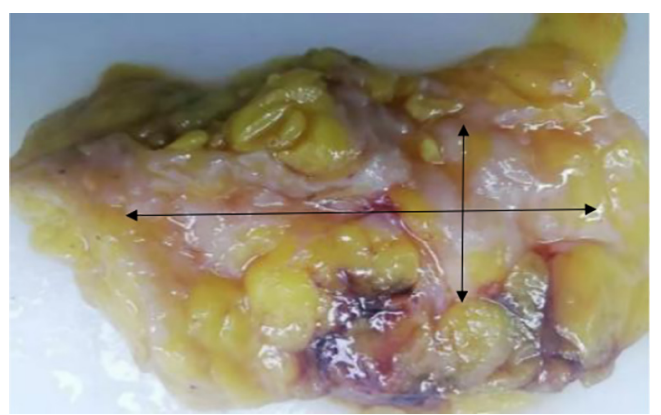

Figure 1 Exposing the largest two-dimensional surface of the tumor bed and measuring the largest diameter.

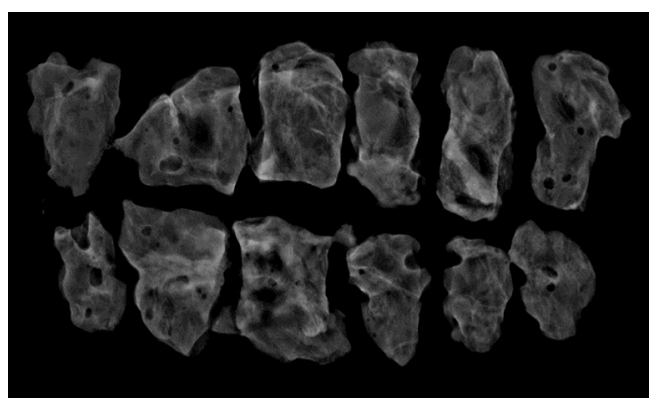

Figure 2 Use CXS to restore the largest two-dimensional surface of the tumor bed. CXS, cabinet X-ray system.

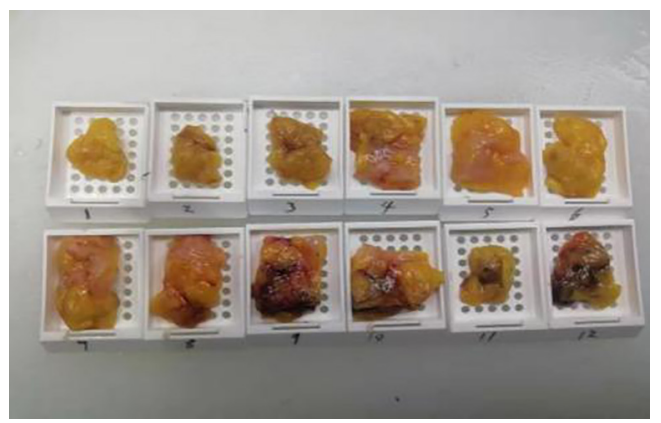

Figure 3 Take the largest two-dimensional surface in sequence from top to bottom, left to right (corresponding to Figures 4,5).

five representative complete samplings of the largest crosssection. You can select a few more of these maximum cross-sections every $1 \mathrm{~cm}$ to determine the full range of the tumor, and record the maximum two-dimensional surface value of the tumor bed. Using a ruler to mark the maximum diameter value, and select the largest diameter of the largest tumor bed in these sections as a reference (Figure 1). At the same time, restore a tumor bed to the

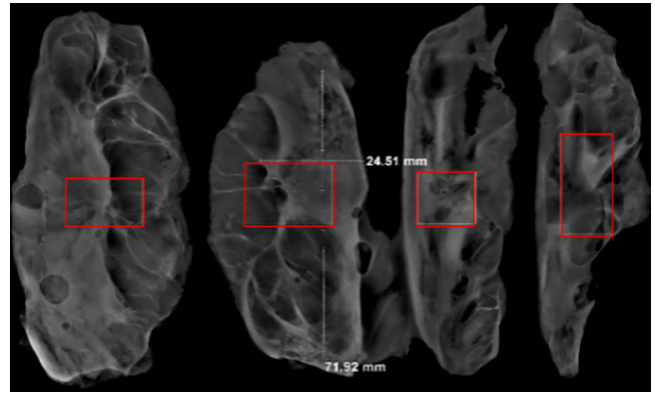

Figure 4 CXS can mark suspicious tumor areas and measure maximum diameter. CXS, cabinet X-ray system.

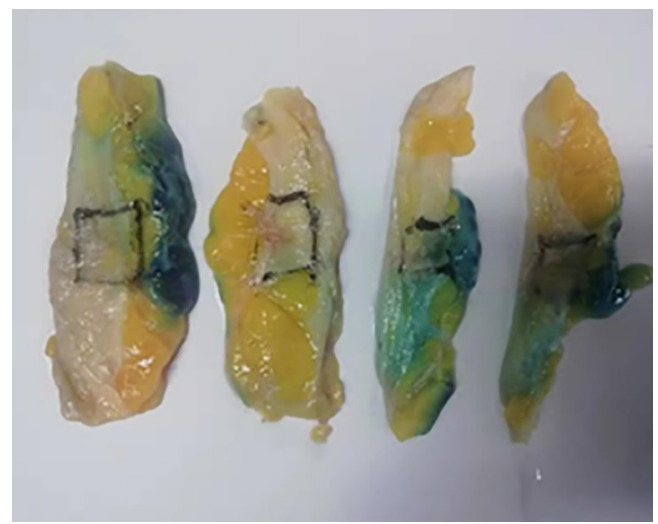

Figure 5 According to the tumor area shown by CXS, multiple sections are marked with corresponding ink stains. CXS, cabinet $\mathrm{X}$-ray system.

largest (Figure 2), then take the largest tumor bed in order and record the corresponding numbers of tissue blocks (Figure 3). To save time, multiple sections can be illuminated together. Use the CXS marking function to mark the area of suspicious tumor beds on each section (Figure 4). Use a marker pen to draw the corresponding suspicious area on the cut surface of the real specimen (Figure 5). Then all the suspected tumors were taken from the area, and the total number of tumor bed materials was recorded.

The tissue block is dehydrated, embedded in paraffin, sectioned and stained with hematoxylin and eosin (HE), and submitted to the experienced pathologist to evaluate the two dimensions of the largest tumor bed section under the microscope, H1, H2 (maximum H1, followed by H2), which needs to be pointed out. $\mathrm{H} 1 / \mathrm{H} 2$ can be obtained by the following methods: observe the farthest end of the tumor under a microscope, mark the glass with black dots, and finally superimpose the glass pieces together and measure 


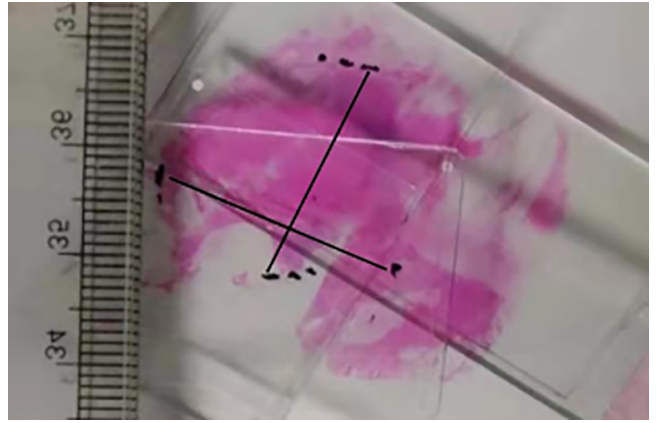

Figure 6 Overlay slices and measure.

the maximum (Figure 6). The two values obtained by the correction under the microscope are as follows: the first one, there is a small error between the macro description and the maximum diameter under the microscope; the second, there is a large discrepancy between the macro value and the final value under the microscope, which is far from above/below final measurement. The determination of the value under the microscope is based on the consensus given by MD Anderson, that is, the largest path through the tumor parenchyma, including non-tumor areas such as fibers, inflammation, and foam cell responses within the measurement. A simple and effective method is to stack the slides to restore the largest two-dimensional surface of the tumor bed, mark both ends of the parenchyma of the tumor at the same time, and measure the two largest diameters in the vertical direction with a ruler. When the tumor bed is large and the parenchymal component of the tumor is small, the complicated workload can be avoided.

\section{Evaluation method based on residual cancer burden (RCB)}

Through the above-mentioned specimen collection and observation under the microscope, we had collected the following data: (I) corrected length under microscope in two vertical directions of the largest two-dimensional surface of tumor bed (d1/d2); (II) percentage of residual tumor (CA\%) and residual ductal carcinoma in situ (DCIS\%) (calculate the value of each slice and get the average); (III) the number of positive lymph nodes and the largest diameter of metastases (dmet) (the measurement of the largest diameter should include the fibrous matrix between the two most distant lesions).

The calculation of the score can use the formula: RCB = 1.4(finv $\times$ dprim $) 0.17+[4(1-0.75 \mathrm{LN}) \mathrm{dmet}] 0.17$, dprim $=$ $\sqrt{ } \mathrm{d} 1 \mathrm{~d} 2$, finv $=[1-(\mathrm{DCIS} \% / 100)] \times(\mathrm{CA} \% / 100) . \mathrm{LN}=$ the number of metastatic lymph nodes.

We log on to http://www.mdanderson.org/breastcancerrcb, enter the above data to calculate the score and get the RCB rating. The scores correspond to different RCB ratings in the following ranges:

* RCB 0: pathologic complete response (pCR);

* RCB I: low risk (0-1.36);

* RCB II: moderate risk (1.37-3.28);

* RCB III: high risk (>3.28).

\section{Statistical analysis}

The number of effective tumor bed materials and the number of tumor bed materials for each case of the experimental group and the control group were recorded. According to the formula: $\mathrm{P}=\mathrm{T} / \mathrm{N} \times 100 \%$, the efficiency of the remaining bed material was calculated. Two independent samples were used. Parametric rank sum test (Wilcoxon W) and the use of SPSS16.0 software to compare the differences between the experimental group and the control group. Record the macroscopic value D1/D2, the CXS value $\mathrm{d} 1 / \mathrm{d} 2$, and the microscopic value $\mathrm{H} 1 / \mathrm{H} 2$ of each case, and calculate the absolute value of the difference from the microscopic value in each dimension: experimental group D1 - H1, D2 - H2, control group d1 - H1, d2 - H2. The nonparametric rank sum test (Wilcoxon W) of two paired samples was used to compare D1 - H1 and d1 - H1; whether there was a difference between D2 - H2 and $\mathrm{d} 2$ $\mathrm{H} 2, \mathrm{P}<0.05$, the difference was statistically significant.

\section{Results}

In 100 cases, 21 cases were evaluated as mild treatment response, 15 cases were moderate treatment response, and 64 cases were severe treatment response. Forty cases were diagnosed with invasive ductal carcinoma of the breast (31 cases with histological grade 2, grade 3 in 9 cases). The statistical results show that the effective number of tumor bed samples taken in the experimental group was 11.8 (median 12.0), the number of tumor bed samples taken was 16.4 (median 15.5), and the average effective sample retention rate was $72.0 \%$ (median $75.0 \%$ ); The effective number of tumor bed materials in control group was 7.5 (median 7.0), the number of tumor bed materials was 16.7 (median 17.0), and the total effective bed retention rate was $44.8 \%$ (median $45.0 \%$ ), There were differences in the effective number of tumor bed materials between the experimental group and the control group $(\mathrm{P}<0.05)$. There 
Table 1 Comparison of T, N, P values between experimental and control groups

\begin{tabular}{lcccc}
\hline \multirow{2}{*}{ Variable } & \multicolumn{2}{c}{ Mean (median) } & Z & Artificial $(\mathrm{n}=50)$ \\
\cline { 2 - 4 } & $\mathrm{CXS}(\mathrm{n}=50)$ & $7.5(7.0)$ & -5.249 & 0.000 \\
$\mathrm{n} \mathrm{T}$ & $11.8(12.0)$ & $16.7(17.0)$ & -0.432 & 0.666 \\
$\mathrm{~N}$ & $16.4(15.5)$ & $44.8(45.0)$ & -7.509 & 0.000 \\
\hline
\end{tabular}

$\mathrm{T}$ : the number of effective specimens; $\mathrm{N}$ : the number of tumor bed specimens; P: the efficiency of retaining bed material (\%). CXS, cabinet X-ray system.

Table 2 Statistical description of macro measurements and CXS measurements ( $\mathrm{mm}$ )

\begin{tabular}{|c|c|c|c|c|c|c|c|c|}
\hline Variables & $\mathrm{N}$ & Mean & Std. deviation & Min & Max & \multicolumn{3}{|c|}{ Percentile } \\
\hline D1 & 100 & 27.7700 & 15.32863 & 4.00 & 100.00 & 20.0000 & 25.0000 & 35.0000 \\
\hline d1 & 100 & 43.7346 & 27.49250 & 8.50 & 134.74 & 24.7825 & 35.1650 & 55.3275 \\
\hline D2 & 100 & 21.1800 & 11.74767 & 3.00 & 80.00 & 15.0000 & 20.0000 & 25.0000 \\
\hline
\end{tabular}

D1: maximum length diameter of the largest surface of the tumor bed measured by eyes; D2: maximum short diameter of the largest surface of the tumor bed measured by eyes; d1: maximum length diameter of the largest surface of the tumor bed measured by CXS; d2: maximum short diameter of the largest surface of the tumor bed measured by CXS. CXS, cabinet X-ray system.

Table 3 Comparison of error value between experimental group and control group

\begin{tabular}{lcc}
\hline Test statistic $^{\mathrm{b}}$ & $\mathrm{d} 1-\mathrm{D} 1$ & $\mathrm{~d} 2-\mathrm{D} 2$ \\
\hline $\mathrm{Z}$ & $-5.770^{\mathrm{a}}$ & $-1.761^{\mathrm{a}}$ \\
Asymp. Sig. (two-tailed) & 0.000 & 0.078 \\
\hline
\end{tabular}

${ }^{a}$, based on negative ranks; ${ }^{b}$, Wilcoxon signed ranks test. D1: maximum length diameter of the largest surface of the tumor bed measured by eyes; D2: maximum short diameter of the largest surface of the tumor bed measured by eyes; d1: maximum length diameter of the largest surface of the tumor bed measured by CXS; d2: maximum short diameter of the largest surface of the tumor bed measured by CXS. CXS, cabinet X-ray system.

was a statistically significant difference in the effective sample retention rate between the experimental group and the control group $(\mathrm{P}<0.05$, Table 1). Macroscopic measurement of the largest two-dimensional surface of the tumor bed D1 average $27.77 \mathrm{~mm}$ (median $25.00 \mathrm{~mm}$ ), D2 average of $21.18 \mathrm{~mm}$ (median $20.00 \mathrm{~mm}$ ); CXS measurement of the largest two-dimensional surface of the tumor bed d1 average $43.73 \mathrm{~mm}$ (median $35.16 \mathrm{~mm}$ ), the average value of $\mathrm{d} 2$ is $21.98 \mathrm{~mm}$ (median $21.49 \mathrm{~mm}$ )
(Table 2). There was a statistical difference between D1 and $\mathrm{d} 1(\mathrm{P}<0.05$, Table 3$)$. There was a statistical difference between D1 - H1 and $\mathrm{d} 1-\mathrm{H} 1, \mathrm{D} 2-\mathrm{H} 2$ and $\mathrm{d} 2-\mathrm{H} 2$ $(\mathrm{P}<0.05$, Tables 4,5$)$. The line chart shows that the measured value of the tumor bed CXS is closer to the measured value of the microscope than the measured value of the macro tumor bed. Using CXS measurement, its error value does not change significantly (Figures 7,8). D1 and d1 was statistically different, which show that the two methods had a measure of the tumor bed without comparing the true values after correction under the microscope was different; $\mathrm{D} 2-\mathrm{H} 2$ and $\mathrm{d} 2-\mathrm{H} 2$ was different, but D2 and $\mathrm{d} 2$ was not different, which indicated that when the tumor bed had a small range, the CXS measurement value was closer to the curve of the measurement value under the microscope, and macro measurement values was far away from the CXS measurement value and the CXS measurement value on the same side of the CXS measurement value curve (macro measurements ware too low or too high).

\section{Discussion}

The treatment of breast cancer has gradually developed 
Table 4 Statistical description of experimental group error value and control group error value $(\mathrm{mm})$

\begin{tabular}{|c|c|c|c|c|c|c|c|c|}
\hline Variable & $\mathrm{N}$ & Mean & Std. deviation & Min & Max & \multicolumn{3}{|c|}{ Percentile } \\
\hline $\mathrm{D} 1-\mathrm{H} 1$ & 100 & 16.8560 & 17.55233 & 0.50 & 75.20 & 4.3000 & 8.1500 & 24.1500 \\
\hline $\mathrm{d} 1-\mathrm{H} 1$ & 100 & 3.8118 & 5.33677 & 0.20 & 34.54 & 1.1000 & 2.1650 & 4.5150 \\
\hline $\mathrm{D} 2-\mathrm{H} 2$ & 100 & 7.4210 & 8.22740 & 0.20 & 61.70 & 2.6000 & 4.4500 & 9.1000 \\
\hline
\end{tabular}

D1: maximum length diameter of the largest surface of the tumor bed measured by eyes; D2: maximum short diameter of the largest surface of the tumor bed measured by eyes; d1: maximum length diameter of the largest surface of the tumor bed measured by CXS; d2: maximum short diameter of the largest surface of the tumor bed measured by CXS; H1: maximum length diameter of the largest surface of the tumor bed measured by microscope; H2: maximum short diameter of the largest surface of the tumor bed measured by microscope. CXS, cabinet X-ray system.

Table 5 Comparison of the difference between the reference experimental group and the control group

\begin{tabular}{lcc}
\hline Test statistic $^{\mathrm{b}}$ & $(\mathrm{D} 1-\mathrm{H} 1)-(\mathrm{d} 1-\mathrm{H} 1)$ & $(\mathrm{D} 2-\mathrm{H} 2)-(\mathrm{d} 2-\mathrm{H} 2)$ \\
\hline $\mathrm{Z}$ & $-8.682^{\mathrm{a}}$ & $-7.961^{\mathrm{a}}$ \\
Asymp. Sig. & 0.000 & 0.000 \\
(two-tailed) & & \\
\hline
\end{tabular}

${ }^{a}$, based on negative ranks; ${ }^{b}$, Wilcoxon signed ranks test. D1: maximum length diameter of the largest surface of the tumor bed measured by eyes; D2: maximum short diameter of the largest surface of the tumor bed measured by eyes; d1: maximum length diameter of the largest surface of the tumor bed measured by CXS; d2: maximum short diameter of the largest surface of the tumor bed measured by CXS; H1: maximum length diameter of the largest surface of the tumor bed measured by microscope; H2: maximum short diameter of the largest surface of the tumor bed measured by microscope. CXS, cabinet X-ray system.

from the initial simple surgical treatment to the current comprehensive treatment plan with surgery as the center, supplemented by chemotherapy, radiotherapy, immunotherapy, endocrine therapy, etc. This is breast cancer research in imaging, surgery, The inevitable result of multidisciplinary comprehensive development such as pathology (1). NACT not only benefits more breast cancer patients, but also brings many problems and challenges to pathologists (2). Specimen selection and evaluation of important pathological parameters are critical to assessing the extent of treatment response, so it is important to recognize that histopathologists play a key role in this multidisciplinary environment. However, there is no uniform standard for the selection of tumor bed materials. This means that taking common methods for breast tumor beds after neoadjuvant may lose important parameters and cause great errors in the RCB score $(3,4)$. According to the latest American Joint Committee on Cancer (AJCC) classification, taking macro- and micro-tissues to evaluate the size range (ypT) of residual cancer after neoadjuvant treatment of the tumor bed is the best combination. After neoadjuvant therapy, tumor bed changes can be roughly divided into two types: concentric contraction; non-concentric contraction (Figures 9,10). Concentric contractions are more common in HER2 overexpression and triple-negative or basal-like types, while non-concentric contractions are mostly luminal breast cancer. When the chemotherapy effect is good, the fibrosis and necrosis often occur in the tumor bed area, and it is often difficult to distinguish the existence of the parenchymal component of the tumor with eyes. Therefore, when the non-concentric contraction tumor bed approaches/reaches complete remission, it is difficult for pathologists to choose materials because they are easy to miss.

According to BIG-NABCG recommendations, when residual cancer is found, a complete cross-section of the largest tumor area should be evaluated under a microscope section. For larger tumors, five representative sections are selected for complete sampling of the largest cross section. Several more such maximum cross-sections can be selected every $1 \mathrm{~cm}$ apart to determine the full extent of the tumor. This method is sufficient to assess the tumor size and the percentage of residual cancer calculated by the AJCC stage and RCB. Correlation with preoperative imaging should be used to help locate the tumor site, and if possible, specimen radiographs should be used to locate the tumorrelated site and/or calcifications before surgery. The size of the tumor before sampling will determine the scope of sampling. Systematic material extraction is preferred, rather than blind material extraction of the entire fiberized area 


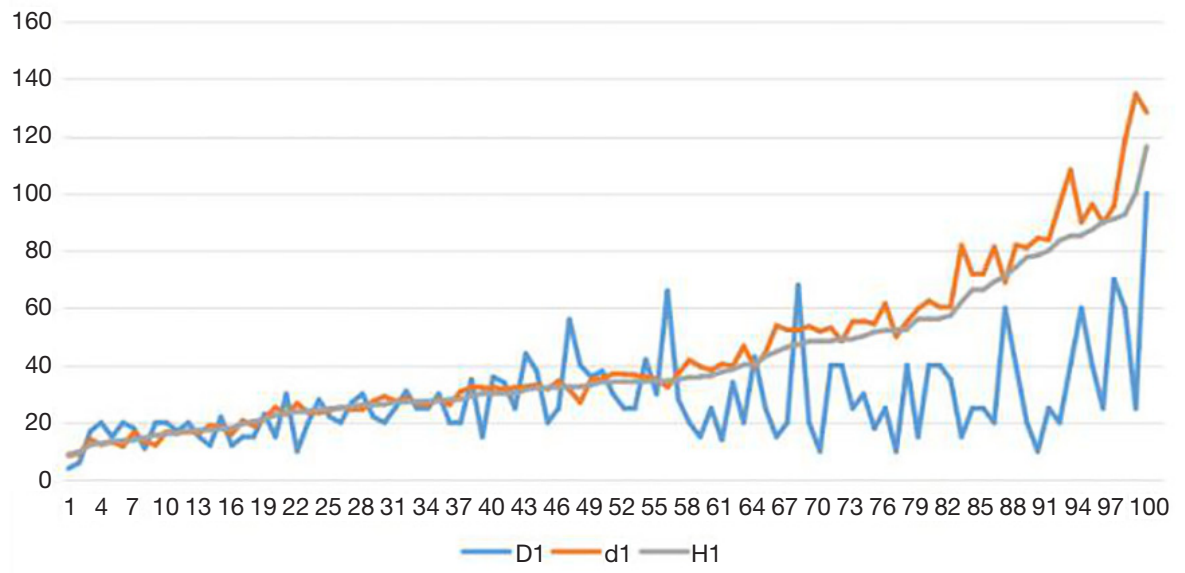

Figure 7 Three measurement methods to measure the maximum two-dimensional surface length of the tumor bed and draw a line chart.

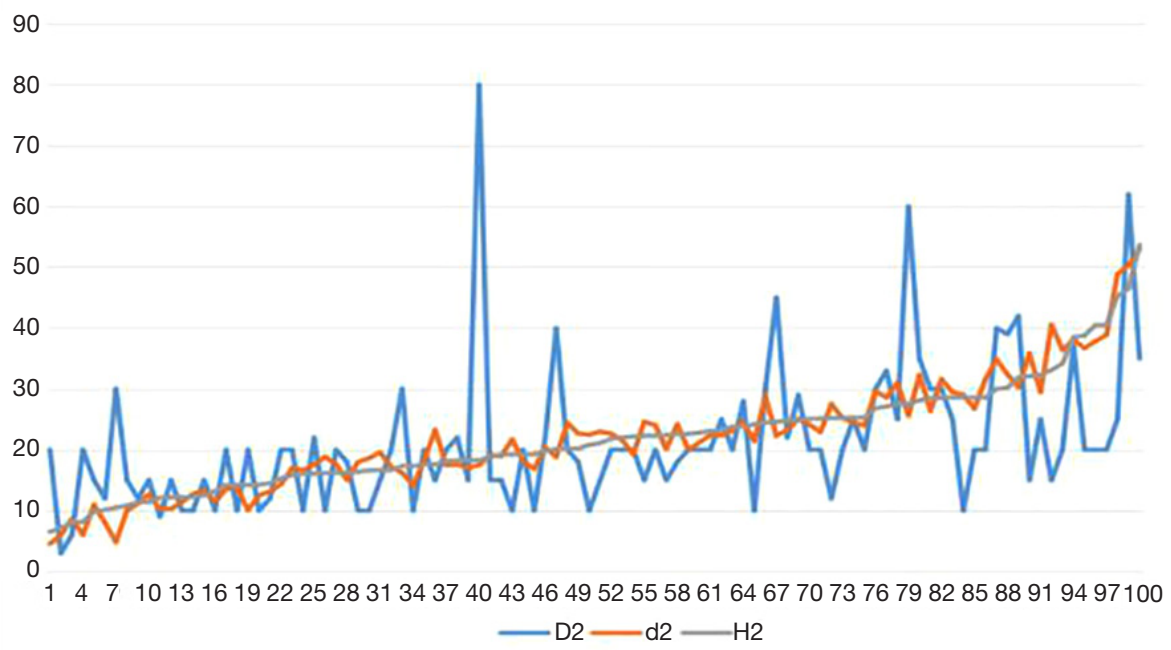

Figure 8 Three measurement methods to measure the shortest diameter of the largest two-dimensional surface of the tumor bed.

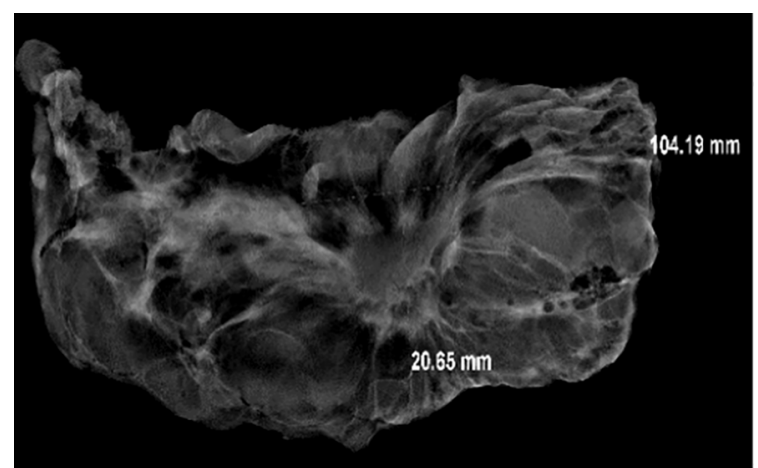

Figure 9 The tumor showed a centripetal atrophy in CXS images. CXS, cabinet X-ray system.

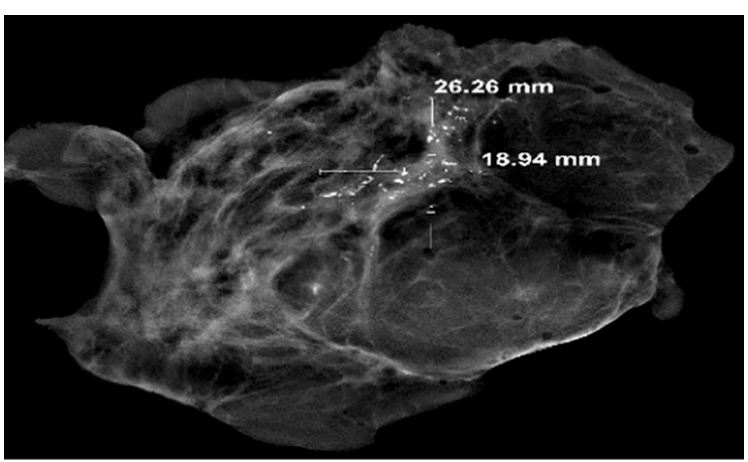

Figure 10 The tumor showed a non-centric atrophy in CXS image. CXS, cabinet X-ray system. 
or any number of pieces. This requires comprehensive judgment through careful study of clinical data and imaging characteristics, so as to select the best area for material extraction. When no tumor residue is found, BIG-NABCG recommends the following method: one largest crosssection [or five representative slices for each $1 \mathrm{~cm}$ (larger tumor bed 1-2 cm)] pretreatment area. For larger tumor beds, take a maximum of 25 . In contrast, the US FDA recommends taking at least one piece per centimeter of the size of the tumor before treatment, or a total of at least ten pieces, whichever is greater. However, the Royal College of Pathologists (UK) does not support neoadjuvant Any specific recommendations for the treatment of specimens are given. For multifocal tumors, each lesion should be treated in the same way, and sections of breast tissue between tumors should be recorded and taken. Obviously, whether it is postoperative Miller-Payne system or RCB system evaluation, the sampling method suggested by BIG-NABCG has the advantages of evaluation. The key to pathological material acquisition is to determine the location of the tumor bed after surgery. If imaging is used as a reference, the omission of the lesion can be avoided to the greatest extent.

In recent years, multidisciplinary cooperation and the rise of artificial intelligence technology have undoubtedly provided better methods for breast cancer screening and diagnosis. For example, using computer-aided diagnosiscontrast enhanced spectral mammography (CAD-CESM) tools, Patel et al. and other studies have found that in the observation of 50 breast cancer patients, CAD CESM correctly identified 45 of the 50 lesions in the cohort with an accuracy of $90 \%$ (5). In the field of breast cancer, the current artificial intelligence technology is mainly used in early imaging screening (6-8), and pathologists use virtual microscopes and remote pathological digital section consultations (9). Maeda and other studies used 200 hollow-core needle biopsy breast specimens to perform immunohistochemical staining on their estrogen receptor (ER), synaptophysin, and CK14/p63, scan the whole section, and analyze the nuclear and cytoplasmic staining with image analysis software as proof of diagnosis cases with high ER expression may indicate malignancy (10).

Preoperative assessment of the patient's tumor bed can use methods such as mammography, magnetic resonance imaging (MRI) and Doppler ultrasound images. The X-ray manifestations of breast cancer are divided into 4 kinds: (I) mass; (II) calcification; (III) structural distortion; (IV) asymmetric dense shadow. The imaging principle is based on the difference between the density of the lesion and the surrounding normal tissues. Therefore, when the treatment effect is not good, high-density lesions are still present. At this time, malignant cells at the edge of the lesion infiltrate the surrounding area, and the normal tissue inflammatory response prevents its development and forms wrapping and pulling, X-ray images show burrs, roughness and lobes; when there is a certain effect, the density of the lesion is reduced and the volume becomes smaller; when the effect is good, the density of the lesion is consistent with the surrounding glands, and the shape is unclear.

MRI is the most sensitive breast cancer detection method and the most accurate imaging method for evaluating the efficacy of neoadjuvant therapy for breast cancer (11). It includes diffusion-weighted imaging, dynamic enhanced MRI, magnetic resonance spectroscopy imaging and other technologies. Compared with clinical evaluation, MRI can more accurately predict the pathological response of breast cancer after neoadjuvant treatment, and the change of tumor volume is more valuable for the evaluation of curative effect than the diameter (12). The treatment response varies with the MRI appearance of the tumor and the subtype of the tumor. MRI showed clear boundaries, triple-negative breast cancer NACT, the consistency of MRI and clinicopathological judgments of tumor size was better than that of MRI interval scatter-like enhancement, hormone receptor $(\mathrm{HR})^{+}$breast cancer (13). At the same time, the type of chemotherapy regimen affects the efficacy of MRI evaluation accuracy $(14,15)$.

Color Doppler ultrasound technology can intuitively evaluate the therapeutic effect, usually with the nipple as the center to do a spoke-like scan, adjust the depth, gain, and focus position according to the actual condition of the mass to ensure a clear image. At the same time, it can also be combined with two-dimensional ultrasound to detect the location, size, boundary, shape, internal echo, etc. of the lesion, and use color Doppler ultrasound mode to observe the blood flow inside the tumor. Refer to the Alder grading standard for grading assessment: level 0 , inside the tumor no blood flow signal; level I, less blood flow signal, and 1 to 2 punctate blood flow with a diameter of less than $1 \mathrm{~mm}$ can be seen; level II, blood flow signal is more obvious, with 3 to 4 blood vessels visible, radially distribution, at least one blood vessel straddles the lesion; level III, the blood flow signal is abundant, and more than 4 blood vessels are visible, which are distributed in a network. Judging the effect of chemotherapy by observing the patient's blood flow resistance index and comparing it with pre-operative 


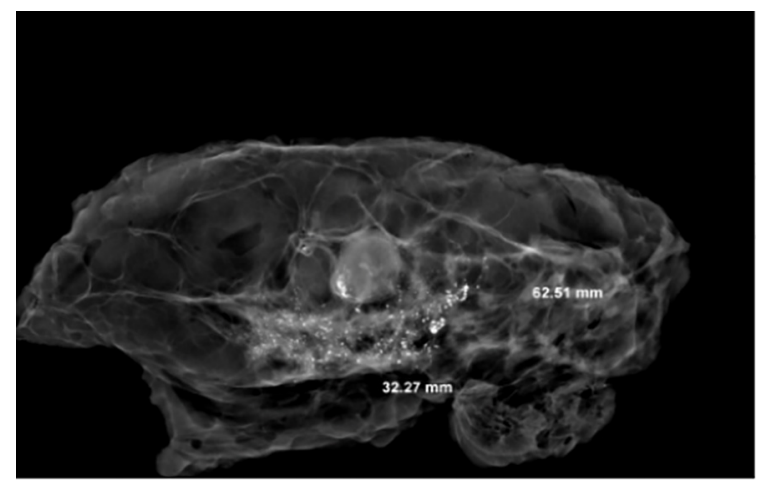

Figure 11 Image of CXS, the largest cross section of the tumor bed shows the strongest signal intensity of invasive cancer and carcinoma in situ. CXS, cabinet X-ray system.

images. If patients are sensitive to chemotherapy drugs, their two-dimensional ultrasound performance, blood flow classification and resistance index will have obvious changes, but there will be no obvious changes when the treatment is ineffective. This is mainly because when the patient is sensitive to chemotherapy drugs, the tumor cells are gradually destroyed, causing the tumor to gradually shrink or even disappear. At the same time, the blood vessels inside the tumor are embolized, collapsed, and occluded, which leads to a decrease in blood flow signals inside the tumor (16).

In this experiment, the CXS can clearly show the suspected area of the tumor bed, and can accurately measure and mark the tumor bed range. CXS includes six main originals. Lead room: composed of two layers of stainlesssteel plates and $6-8-\mathrm{mm}$ thick lead plates; stage: used to place samples; detector: used to sense $\mathrm{X}$-ray intensity and generate black and white signals to the computer; tube: generate X-ray; high voltage generator: generate $160 \mathrm{kV}$ voltage to supply power to the entire system; vacuum system: X-ray tube must work in vacuum. Operation steps: after the power is turned on, the high-voltage generator generates high voltage and acts on the tube to generate tube current (total number of electrons escaping from the filament), including tube wall current and target current (the actual number of electrons reaching the target). The actual area of the target that the target current hits on the $\mathrm{X}$-ray tube is called the focal point size, which determines the detection of the smallest defect of the object. Generally speaking, the smaller the focal point, the smaller the defect detected. The $\mathrm{X}$-rays generated by the target current acting on the target can illuminate the sample and then image it on a digital camera. When the radiation penetrates the sample and reaches the sensing material, there is a voltage difference of $20 \mathrm{kV}$ between the material and the phosphor screen. Due to the different X-ray intensity, the sensing material will produce different numbers of electrons which are accelerated and hit the phosphor screen to form a black and white photo. The digital camera will take pictures and the photos are converted into digital signals and sent to the computer. We identify tumors by the gray scale of the image from black to white (level 25, blackest to whitest)

On the X-ray image, the tumor bed showed obvious high-density signal areas, and the calcification and DCIS showed brighter light spots (Figure 11). Radially dense white shadowed areas of the tumor bed with radial constriction are clearly distinguished from the surrounding black lowdensity fat area. Non-concentrically contracted tumor beds differ only in signal intensity, but the corresponding range can be determined. The comparison of the sampling efficiency between the experimental group and the control group shows that although there is no statistical difference between the two groups, the effective sampling rate of the total tumor bed in the experimental group is more than that in the control group. The experimental group has a clear advantage. The reason is that eyes is very easy to miss when identifying areas of more obvious treatment response.

The CXS play an important role in the maximum two-dimensional cross section of the tumor bed and the restoration of the tumor bed. When the samples were too small, this situation was more common in breast-conserving samples of neoadjuvant treatment. The determination of the tumor bed range not only help to obtain the largest two-dimensional surface, but also found the edge closest to the tumor. The vertical tumor bed could be used. The direction was taken from the nearest margin. We irradiated the sample with CXS, observed the range of the suspicious density signal of the tumor bed, measured the distance from the tumor bed to the margin and mark it. If there was residual DCIS, it was easier to observe. Because the tumor bed was too small and the boundary was not clear, we took the evaluation of the scope of the microscope as the best guiding principle, and require that the number of pieces (1-2 pieces) on the largest surface when taking talents to increase the accuracy of slice splicing. While taking the smaller tumor bed, the adipose tissue around the tumor bed can be expanded, so as to avoid the area that is not visible to the eyes or CXS from being missed.

In this experiment, the measured value of the tumor bed of the control group and the measured value under 


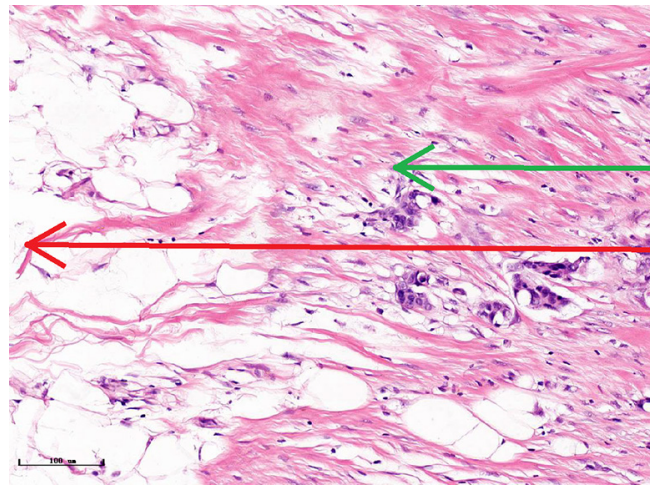

Figure 12 Presence of fibrous reactions at the edge of the tumor bed results in microscopic measurements less than macro/CXS measurements (hematoxylin and eosin). CXS, cabinet X-ray system.

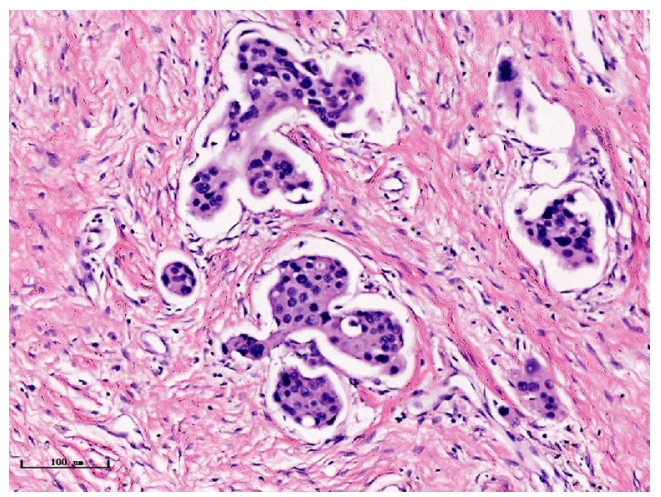

Figure 13 Microscopic infiltrates under the microscope, which are difficult to find by manual measurement (corresponds to the brightest point of the signal in Figure 1) (hematoxylin and eosin).

the microscope have a large error. Compared with the experimental group, the error value is more unstable, and the difference is statistically significant. When the tumor bed is larger, the error in the control group is more obvious, but no obvious change in the experimental group, which proves that when the effect of chemotherapy is good and the tumor parenchyma is not obvious, there is a wide range of suspicious tumor beds. Sometimes, because the dense fibrous response at the edge of the tumor bed is not easy to distinguish from lower density cancerous lesions, the diameter measured at the outermost edge of the tumor parenchyma observed under the microscope is smaller than the macro measurement value (Figure 12), but CXS can solve this problem. CXS can play an accurate

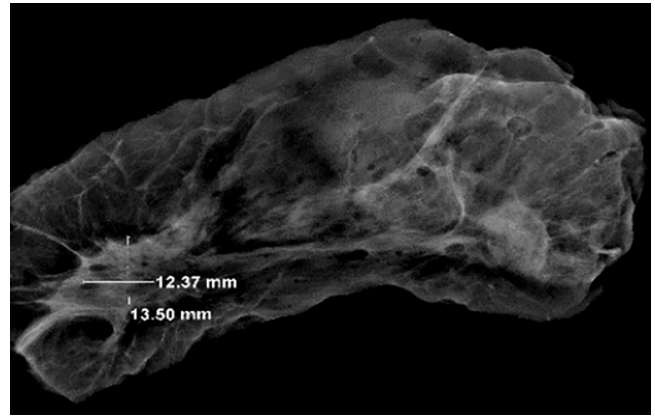

Figure 14 Tumor position shift after neoadjuvant chemotherapy (bottom left).

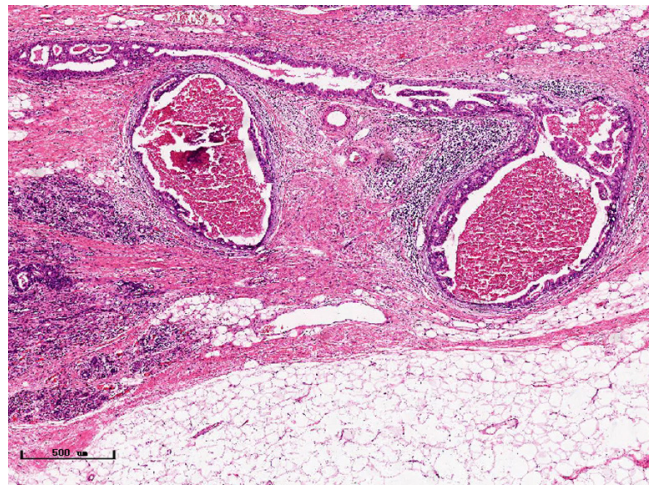

Figure 15 High-luminance points on the X-ray image under microscope are ductal carcinoma in situ remaining from the tumor bed (hematoxylin and eosin).

assessment role, when small focal tumors remain on the edge of the tumor bed, too small lesions are easily missed when the maximum diameter is plotted by the naked eye (Figure 13), and on X-ray imaging, they show strong signal points. Small lesions are therefore detected, which avoids the need for lesions omission. The shift of the tumor's main body position after NACT is also a major factor affecting macro measurement, but CXS images can clearly find this phenomenon (Figure 14). One of the advantages of CXS is that it will appear as invasive cancer, DCIS, microcalcifications, and fibrous fat response under the microscope (Figures 15-17), and distinguish the images by different imaging signal densities.

In summary, in the era of rapid development of medical technology, the emergence of new pathological materials and diagnostic auxiliary equipment represented by artificial intelligence requires us to continuously explore its advantages and explore its potential in order to overcome 


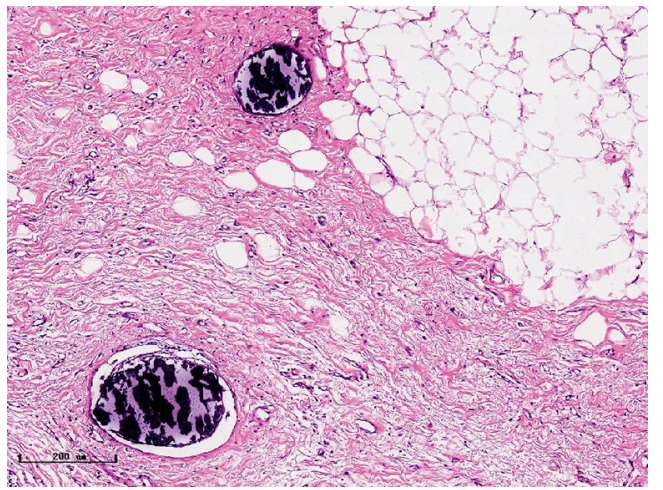

Figure 16 The high-brightness points on the X-ray map can also be calcifications (hematoxylin and eosin).

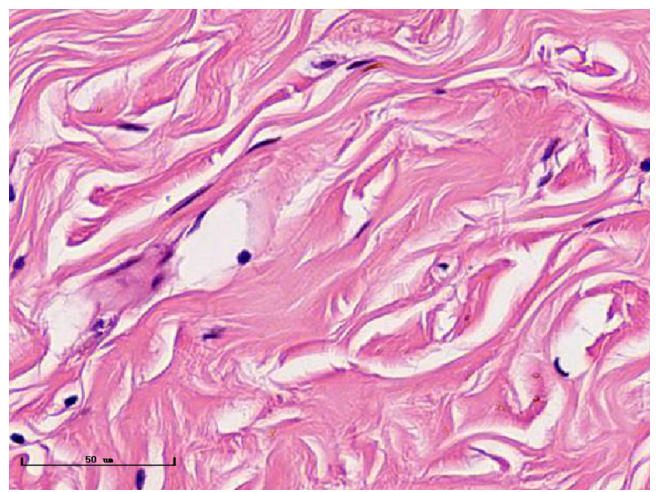

Figure 17 The lower signal areas on the X-ray diagram often appear fibrotic under the microscope (hematoxylin and eosin).

difficulties encountered in daily work. However, the role of the CXS needs to be studied and explored by more scholars.

\section{Acknowledgments}

Funding: None.

\section{Footnote}

Reporting Checklist: The authors have completed the MDAR checklist. Available at http://dx.doi.org/10.21037/ tcr-20-2373

Data Sharing Statement: Available at http://dx.doi. org/10.21037/tcr-20-2373

Conflicts of Interest: All authors have completed the ICMJE uniform disclosure form (available at http://dx.doi. org/10.21037/tcr-20-2373). The authors have no conflicts of interest to declare.

Ethical Statement: The authors are accountable for all aspects of the work in ensuring that questions related to the accuracy or integrity of any part of the work are appropriately investigated and resolved. The study was conducted in accordance with the Declaration of Helsinki (as revised in 2013). The study was approved by ethics board of the Fourth Hospital of Hebei Medical University (2020K1334 ) and informed consent was taken from all the patients.

Open Access Statement: This is an Open Access article distributed in accordance with the Creative Commons Attribution-NonCommercial-NoDerivs 4.0 International License (CC BY-NC-ND 4.0), which permits the noncommercial replication and distribution of the article with the strict proviso that no changes or edits are made and the original work is properly cited (including links to both the formal publication through the relevant DOI and the license). See: https://creativecommons.org/licenses/by-nc-nd/4.0/.

\section{References}

1. Peng JH, Zhang X, Song JL, et al. Neoadjuvant chemotherapy reduces the expression rates of ER, PR, HER2, Ki67, and P53 of invasive ductal carcinoma. Medicine (Baltimore) 2019;98:e13554.

2. Wu NC, Wong W, Ho KE, et al. Comparison of central laboratory assessments of ER, PR, HER2, and Ki67 by IHC/FISH and the corresponding mRNAs (ESR1, PGR, ERBB2, and MKi67) by RT-qPCR on an automated, broadly deployed diagnostic platform. Breast Cancer Res Treat 2018;172:327-38.

3. Chen K, Liu C, Sheng Y. Application of residual tumor burden score in the evaluation of neoadjuvant chemotherapy for breast cancer. Chinese Journal of Breast Disease (Electronic Edition) 2018;12:113-5.

4. Naidoo K, Parham DM, Pinder SE. An audit of residual cancer burden reproducibility in a UK context. Histopathology 2017;70:217-22.

5. Patel BK, Ranjbar S, Wu T, et al. Computer-aided diagnosis of contrast-enhanced spectral mammography: A feasibility study. Eur J Radiol 2018;98:207-13.

6. Houssami N, Kirkpatrick-Jones G, Noguchi N, Lee CI. Artificial Intelligence (AI) for the early detection of breast cancer: a scoping review to assess AI's potential in breast screening practice. Expert Rev Med Devices 
2019;16:351-62.

7. Rodriguez-Ruiz A, Lång K, Gubern-Merida A, et al. Stand-Alone Artificial Intelligence for Breast Cancer Detection in Mammography: Comparison With 101 Radiologists. J Natl Cancer Inst 2019;111:916-22.

8. Mani S, Chen Y, Arlinghaus LR, et al. Early prediction of the response of breast tumors to neoadjuvant chemotherapy using quantitative MRI and machine learning. AMIA Annu Symp Proc 2011;2011:868-77.

9. Montalto MC. An industry perspective: An update on the adoption of whole slide imaging. J Pathol Inform 2016;7:18.

10. Maeda I, Kubota M, Ohta J, et al. Effectiveness of computer-aided diagnosis (CADx) of breast pathology using immunohistochemistry results of core needle biopsy samples for synaptophysin, oestrogen receptor and CK14/ p63 for classification of epithelial proliferative lesions of the breast. J Clin Pathol 2017;70:1057-62.

11. Croshaw R, Shapiro-Wright H, Svensson E, et al. Accuracy of clinical examination, digital mammogram, ultrasound, and MRI in determining postneoadjuvant pathologic tumor response in operable breast cancer patients. Ann Surg Oncol 2011;18:3160-3.

Cite this article as: Zhang M, Ma Y, Geng C, Liu Y. Assisted computer and imaging system improve accuracy of breast tumor size assessment after neoadjuvant chemotherapy. Transl Cancer Res 2021;10(3):1346-1357. doi: 10.21037/tcr-20-2373
12. Hylton NM, Blume JD, Bernreuter WK, et al. Locally advanced breast cancer: MR imaging for prediction of response to neoadjuvant chemotherapy--results from ACRIN 6657/I-SPY TRIAL. Radiology 2012;263:663-72.

13. Mukhtar RA, Yau C, Rosen M, et al. Clinically meaningful tumor reduction rates vary by prechemotherapy MRI phenotype and tumor subtype in the I-SPY 1 TRIAL (CALGB 150007/150012; ACRIN 6657). Ann Surg Oncol 2013;20:3823-30.

14. Denis F, Desbiez-Bourcier AV, Chapiron C, et al. Contrast enhanced magnetic resonance imaging underestimates residual disease following neoadjuvant docetaxel based chemotherapy for breast cancer. Eur J Surg Oncol 2004;30:1069-76.

15. Bahri S, Chen JH, Mehta RS, et al. Residual breast cancer diagnosed by MRI in patients receiving neoadjuvant chemotherapy with and without bevacizumab. Ann Surg Oncol 2009;16:1619-28.

16. Nurcahyanti AD, Wink M. L-Canavanine Potentiates Cytotoxicity of Chemotherapeutic Drugs in Human Breast Cancer Cells. Anticancer Agents Med Chem 2017;17:206-11. 